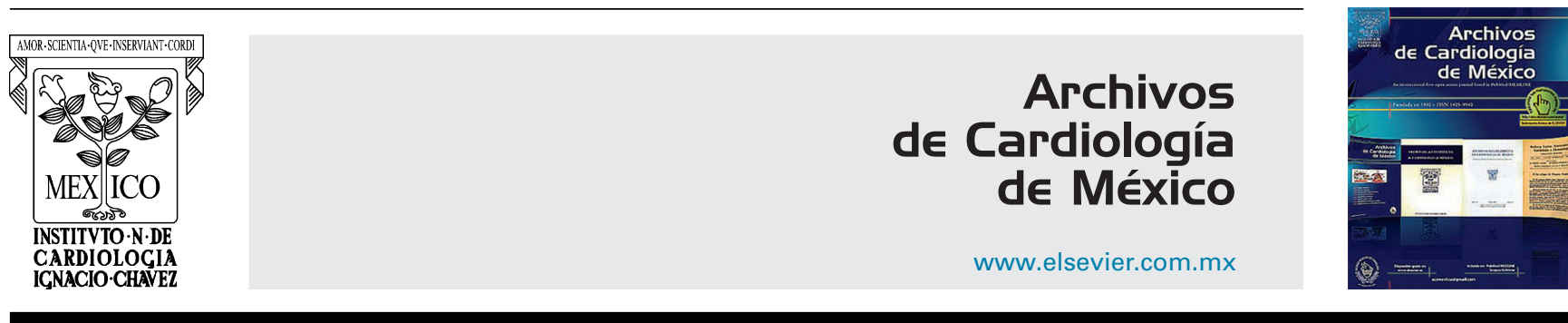

INVESTIGACIÓN CLÍNICA

\title{
Determinación del valor pronóstico a 6 meses del puntaje de riesgo OESIL en una cohorte colombiana con síncope evaluada en el servicio de urgencias, primera experiencia latinoamericana
}

\author{
Diana Carolina Díaz-Tribaldos ${ }^{a}$, Guillermo Mora ${ }^{\mathrm{a}, \mathrm{b}, \mathrm{c}, *}$, Alejandro Olaya ${ }^{\mathrm{b}, \mathrm{d}, \mathrm{e}}$, \\ Jorge Marín ${ }^{f}$ y Fabio Sierra Matamoros ${ }^{\mathrm{g}}$
}

a Departamento de Medicina Interna, Facultad de Medicina, Universidad Nacional de Colombia, Bogotá, Colombia

b Servicio de Electrofisiología, Fundación Santa Fe de Bogotá, Bogotá, Colombia

c Servicio de Electrofisiología, Hospital Universitario Clínica San Rafael, Bogotá, Colombia

d Servicio de Electrofisiología, Departamento de Cardiología, Hospital de San José, Bogotá, Colombia

e Servicio de Cardiología, Fundación Universitaria de Ciencias de la Salud, Bogotá, Colombia

f Servicio de Cardiología, Clínica CES, Medellín, Colombia

` Epidemiología Clínica, Fundación Universitaria de Ciencias de la Salud, Bogotá, Colombia

Recibido el 4 de noviembre de 2016; aceptado el 19 de junio de 2017

\section{PALABRAS CLAVE \\ Síncope; \\ Valor predictivo; \\ Sensibilidad; \\ Especificidad; \\ Colombia}

\begin{abstract}
Resumen
Objetivos: Determinar el rendimiento pronóstico con sensibilidad, especificidad, valor predictivo positivo y valor predictivo negativo del puntaje de riesgo en síncope OESIL para la predicción de eventos graves (mortalidad, intervenciones invasivas cardiovasculares y reingresos) a 6 meses en adultos que ingresan a urgencias por eventos de síncope.

Métodos: Estudio de cohorte, prospectivo y multicéntrico, con selección de personas mayores de 18 años que ingresaron a urgencias con síncope como motivo de consulta. Se tomaron datos demográficos y clínicos de los participantes, se aplicó el puntaje de riesgo OESIL y para la documentación de eventos graves, se hizo seguimiento por 6 meses a través de contacto telefónico.

Resultados: Se incluyeron 161 pacientes. Una puntuación OESIL de 2 o más puntos se consideró de alto riesgo y se presentó en el $72 \%$ de los casos. Con un corte de 2 puntos o más, las características del puntaje de riesgo OESIL para predecir el desenlace compuesto de mortalidad, intervenciones y reingresos fueron de 75.7, 30.5, 43.1, y 64.4\% para sensibilidad, especificidad, valor predictivo positivo y valor predictivo negativo, respectivamente.
\end{abstract}

* Autor para correspondencia. Departamento de Medicina Interna Of. 501, Facultad de Medicina, Ciudad Universitaria, Bogotá (Colombia). Teléfono y fax: +3165000 . Ext. 15011.

Correo electrónico: gmorap@unal.edu.co (G. Mora). 


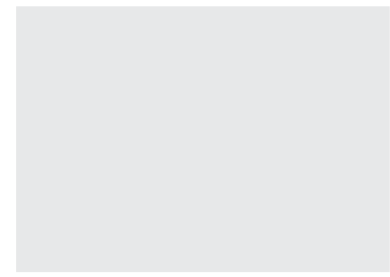

\section{KEYWORDS}

Syncope;

Predictive value;

Sensitivity;

Specificity;

Colombia
Conclusiones: Una puntuación de 2 o más en el puntaje de riesgo OESIL aplicada a población colombiana tiene un desempeño global limitado para la predicción de eventos graves, que no permitiría una adecuada discriminación de los pacientes en riesgo que se benefician de ingreso hospitalario y estudios adicionales.

(C) 2017 Instituto Nacional de Cardiología Ignacio Chávez. Publicado por Masson Doyma México S.A. Este es un artículo Open Access bajo la licencia CC BY-NC-ND (http://creativecommons. org/licenses/by-nc-nd/4.0/).

Determination of prognostic value of the OESIL risk score at 6 months in a Colombian cohort with syncope evaluated in the emergency department; first Latin American experience

\begin{abstract}
Objectives: To establish the prognostic value, with sensitivity, specificity, positive predictive value, and negative predictive value for the OESIL syncope risk score to predict the presentation of severe outcomes (death, invasive interventions, and readmission) after 6 months of observation in adults who consulted the emergency department due to syncope.

Methods: Observational, prospective, and multicentre study with enrolment of subjects older than 18 years, who consulted in the emergency department due to syncope. A record was mad of the demographic and clinical information of all patients. The OESIL risk score was calculated, and severe patient outcomes were followed up during a 6 month period using telephone contact. Results: A total of 161 patients met the inclusion criteria and were followed up for 6 months. A score above or equal to 2 in the risk score, classified as high risk, was present in $72 \%$ of the patients. The characteristics of the risk score to predict the combined outcome of mortality, invasive interventions, and readmission for a score above or equal to 2 were $75.7,30.5,43.1$, and $64.4 \%$ for sensitivity, specificity, positive predictive value, and negative predictive value, respectively.

Conclusions: A score above or equal to 2 in the OESIL risk score applied in Colombian population was of limited use to predict the studied severe outcomes. This score will be unable to discriminate between patients that benefit of early admission and further clinical studies.

(c) 2017 Instituto Nacional de Cardiología Ignacio Chávez. Published by Masson Doyma México S.A. This is an open access article under the CC BY-NC-ND license (http://creativecommons. org/licenses/by-nc-nd/4.0/).
\end{abstract}

\section{Introducción}

El síncope se define como la pérdida transitoria de la conciencia secundaria a un estado de hipoperfusión cerebral, caracterizada por un inicio rápido, corta duración y la recuperación espontánea y completa del estado de conciencia ${ }^{1}$. $\mathrm{Si}$ bien es un motivo de consulta frecuente en los servicios de urgencias $(1-4 \%)^{2,3}$, la gran mayoría de las veces tiene una etiología benigna subyacente. Sin embargo, hasta en un $20 \%$ de los casos es la manifestación de una enfermedad potencialmente mortal, generalmente de origen cardiovascular ${ }^{4,5}$.

Se convierte en un reto para el clínico del servicio de urgencias decidir a qué pacientes debe ingresar para observación y estudios adicionales, sin generar hospitalizaciones y gastos de atención en salud innecesarios. Se han desarrollado múltiples herramientas con el fin de predecir el riesgo de mortalidad u otros desenlaces graves, tanto a corto como a largo plazo ${ }^{5}$. A corto plazo se diseñó la escala de San Francisco del $2004^{6}$ con predicción de muerte y eventos graves a 7 días, y las escalas de Boston de $2007^{7}$ y ROSE de $2010^{8}$ con predicciones a un $\operatorname{mes}^{9,10}$. Las escalas a largo plazo determinan el riesgo a uno o 2 años, y entre ellas se encuentra las escalas de Martin Pittsburg ${ }^{1,9}$, EGSYS $^{11,12}$ y Osservatorio Epidemiologico sulla Sincope nel Lazio (OESIL) $)^{5}$. La gran mayoría tienen como inconveniente que no cuentan con validación externa que corrobore los resultados originales ${ }^{13}$. Los pocos grupos de investigación que han aplicado las escalas en grupos poblacionales diferentes a los de los estudios originales han tenido reportes divergentes ${ }^{13}$.

La escala o puntaje de riesgo OESIL determina 4 factores de riesgo para mortalidad y complicación grave por síncope: electrocardiograma alterado (OR 1.29; IC 95\% 1.16-1.43), ausencia de síntomas prodrómicos (OR 1.13; IC 95\% 1.061.21), historia de enfermedad cardiovascular (OR 1.34; IC $95 \% 1.19-1.49$ ) y la edad mayor de 65 años (OR 1.42; IC 95\% 1.24-1.62) $)^{13,14}$.

Latinoamérica no cuenta con estudios formales de validación de ninguna de las escalas de riesgo aplicables a su población; es por eso que se hace necesaria la realización de estudios que determinen tanto la sensibilidad como la especificidad y los valores predictivos para cada una de estas. Este estudio tiene como intención determinar las características operativas del puntaje de riesgo OESIL para predecir mortalidad y eventos graves a 6 meses. 


\section{Metodología}

Se trata de un estudio de cohorte prospectivo y multicéntrico, en el cual se seleccionó a pacientes adultos mayores de 18 años que consultaron por síncope a los servicios de urgencias del Hospital Universitario Clínica San Rafael, el Hospital San José, la Fundación Santa Fe de Bogotá y la Clínica CES de Medellín, entre agosto del 2013 y julio de 2015. El síncope fue definido según el Task Force de la Sociedad Europea de Cardiología de $2009^{1}$.

Previa firma de consentimiento informado por parte de los participantes, se realizó la recogida de información demográfica y clínica mediante entrevista directa y revisión de historias clínicas de los pacientes ingresados a urgencias por síncope en las $48 \mathrm{~h}$ previas. Se tomaron como criterios de inclusión la edad igual o mayor a 18 años, que presentaran síncope según la definición de la Sociedad Europea de Cardiología, que autorizaran su libre participación y que el paciente o su familia comprendieran el idioma español tanto hablado como escrito. Se excluyeron los pacientes que ingresaran por alteración del estado de conciencia en asociación con consumo de alcohol, estados de hipoglucemia o clínica indicativa de accidente cerebrovascular con déficit cognitivo o del lenguaje, historia de epilepsia o trauma craneoencefálico previo a la pérdida de la conciencia, analfabetos y aquellos que expresaran disentimiento de procedimientos invasivos y maniobras de reanimación.

Una vez confirmada la elegibilidad de los pacientes se diligenció el formulario de recogida de datos, donde se documentaron las variables del puntaje de riesgo OESIL: edad > 65 años, antecedente de enfermedad cardiovascular, ausencia de pródromos y evidencia de alteraciones electrocardiográficas; estas últimas, definidas por anormalidades del ritmo, la conducción auriculoventricular, bloqueos de rama, signos de hipertrofia ventricular derecha o izquierda, desviaciones del eje a la izquierda, ondas de isquemia, lesión o necrosis. Para el registro de los posibles desenlaces se realizó seguimiento a los pacientes durante 6 meses, mediante revisión de historias clínicas y contacto telefónico. Se evaluaron como puntos de desenlace: muerte, infarto agudo de miocardio ${ }^{15}$, arritmias sostenidas, reingresos por síncope, intervenciones cardiovasculares invasivas (cateterismo cardiaco, cirugía cardiaca, implante de marcapasos o cardiodesfibriladores, uso de balón de contrapulsación y ablación de arritmias) o eventos neurológicos.

\section{Análisis estadístico de datos}

Teniendo en cuenta que el estudio se realizó para determinar el valor de las características operativas del puntaje de riesgo OESIL aplicado en población colombiana, se calculó un tamaño de la muestra de 161 pacientes. Este cálculo se realizó considerando un error tipo । de 0.05 , un poder del $80 \%$, y una sensibilidad y una especificidad esperadas de 80 y $59 \%$, respectivamente ${ }^{16}$.

Teniendo en cuenta que el estudio se realizó para determinar el valor de las características operativas del puntaje de riesgo OESIL aplicado en población colombiana y los resultados de sensibilidad previamente reportados, se hizo el cálculo del tamaño de la muestra asumiendo una sensibilidad del $90 \%$, un poder del $80 \%$, un error tipo । de $0.05 \mathrm{y}$ un delta de 0.12 . Se siguió el procedimiento propuesto por Flahault et al. ${ }^{16}$ y Chu y Cole $^{17}$ para la determinación del tamaño de la muestra para pruebas diagnósticas y se empleó la librería MKmisc del paquete estadístico R. Se ajustó el tamaño asumiendo un $15 \%$ de pérdidas de seguimiento. De esta manera se consideró un tamaño de muestra de 160 pacientes.

La descripción de las características sociodemográficas y clínicas y los puntajes obtenidos en la escala OESIL se hizo por medio de frecuencias absolutas y relativas. Los eventos adversos (muerte, reingresos hospitalarios, intervenciones invasivas cardiovasculares y eventos neurológicos) fueron descritos por medio de incidencias acumuladas. Los puntajes de OESIL obtenidos fueron resumidos por medio de frecuencias absolutas y relativas.

Para calcular la sensibilidad, la especificidad y los valores predictivos para cada uno de los posibles puntajes de la escala OESIL se construyeron tablas de contingencia y curvas de características operativas del receptor (ROC) (Stata $13^{\circledast}$ y R), de esta manera se determinó también el área bajo la curva para evaluar la capacidad de discriminación de la escala en la predicción de eventos adversos a los 6 meses. Se llevó a cabo un cálculo de estas características para cada uno de los desenlaces a estudio: mortalidad, reingresos y requerimiento de intervenciones invasivas a nivel cardiovascular a los 6 meses. Se calculó un intervalo de confianza del $95 \%$ para cada una de las características operativas.

El estudio se llevó a cabo con la previa aprobación de los comités de ética de cada una de las respectivas instituciones, siguiendo los protocolos vigentes para investigación.

\section{Resultados}

Se incluyeron 161 pacientes que cumplieron los criterios de inclusión y a quienes se realizó seguimiento para definir la presencia de desenlaces graves a 6 meses. Las características sociodemográficas y las comorbilidades de la población se encuentran en la tabla 1 . El $54.6 \%$ de la población era mayor de 65 años y el 34\% presentaba antecedentes de falla cardiaca de diferente etiología, siendo la isquémica la más prevalente, con un $15 \%$, seguida de la cardiopatía valvular, con un $8 \%$. El $11 \%$ de la población presentaba concomitantemente algún tipo de arritmia, 3 de ellos con uso de marcapasos por bradiarritmias sintomáticas. Cuatro participantes tenían antecedente de colocación de cardiodesfibrilador implantable, la mayoría para prevención primaria de muerte súbita, solo uno de ellos con indicación de prevención secundaria.

Al indagar las características del episodio sincopal, se observó que el $60 \%$ de los sujetos presentaron algún síntoma prodrómico, siendo la diaforesis el más común. En el 44.5\% de la población, el episodio sincopal se asoció a disnea y a dolor torácico (tabla 1).

Según los resultados de estudios previos de validación de la escala, se asumió como punto de corte un puntaje de OESIL mayor o igual a 2 para clasificar a los pacientes en bajo $\mathrm{y}$ alto riesgo de eventos adversos. De los pacientes incluidos, 45 (27.95\%) fueron clasificados como de bajo riesgo, con puntajes OESIL de 0 y 1 , y 116 (72.05\%) se clasificaron en alto riesgo, con puntajes de 2 a 4 (tabla 2 ). 
Tabla 1 Características sociodemográficas y clínicas de los participantes en el estudio

\begin{tabular}{|c|c|}
\hline Característica & Descripción \\
\hline Edad (años), mediana (RIC) & $67(50-77)$ \\
\hline \multicolumn{2}{|l|}{ Género, n (\%) } \\
\hline Masculino & $66(40.99)$ \\
\hline Femenino & $95(59.01)$ \\
\hline \multicolumn{2}{|l|}{ Raza, n (\%) } \\
\hline Caucásica & $69(42.85)$ \\
\hline Mestiza & $85(52.79)$ \\
\hline Indígena & $3(1.86)$ \\
\hline Afrodescendiente & $4(2.48)$ \\
\hline \multicolumn{2}{|l|}{ Comorbilidades, n (\%) } \\
\hline Falla cardiaca & $55(34.16)$ \\
\hline Cardiopatía isquémica & $25(15.52)$ \\
\hline Cardiopatía valvular & $13(8.07)$ \\
\hline Cardiopatía congénita & $4(2.48)$ \\
\hline Miocardiopatía dilatada & $8(4.96)$ \\
\hline Enfermedad arterial periférica & $13(8.07)$ \\
\hline Enfermedad cerebrovascular & $9(5.59)$ \\
\hline Enfermedad renal crónica & $13(8.07)$ \\
\hline Arritmias & $18(11.18)$ \\
\hline Usuarios de marcapasos & $3(1.86)$ \\
\hline Usuarios de CDI & $4(2.48)$ \\
\hline \multicolumn{2}{|l|}{ Características del síncope, n (\%) } \\
\hline Disnea & $54(44.54)$ \\
\hline Deshidratación & $3(1.86)$ \\
\hline Hemorragia gastrointestinal & $4(2.48)$ \\
\hline Dolor torácico & $54(44.54)$ \\
\hline Síncope con el esfuerzo & $7(4.34)$ \\
\hline Síncope por calor & $13(9.07)$ \\
\hline $\begin{array}{l}\text { Traumatismo posterior a pérdida de } \\
\text { conciencia }\end{array}$ & $35(21.73)$ \\
\hline Síntomas prodrómicos & $98(60.86)$ \\
\hline Palpitaciones & $23(14.28)$ \\
\hline Visión borrosa & $58(36.02)$ \\
\hline Diaforesis & $60(37.26)$ \\
\hline Náuseas & $45(27.95)$ \\
\hline Piloerección & $18(11.18)$ \\
\hline
\end{tabular}

CDI: cardiodesfibrilador implantable; RIC: rango intercuartílico.

En el seguimiento a 6 meses se cuantificó la incidencia de eventos graves, encontrando 10 casos $(6.21 \%)$ de muerte. Así mismo, se observaron 22 casos de infarto agudo de miocardio (13.66\%), 6 de ellos con desenlace fatal. Se realizaron 50 procedimientos invasivos a nivel cardiovascular en 42 pacientes (26.08\%), en los cuales se incluye la realización de cateterismo cardiaco, la colocación de marcapasos, cardiodesfibriladores implantables y cardiorresincronizadores, revascularización miocárdica, cirugía valvular e intervención por electrofisiología para ablación de focos arritmogénicos. Se debe aclarar que en 6 pacientes se consideró indicación y realización de 2 o más de los procedimientos descritos. En cuanto a eventos neurológicos, se documentaron 7 accidentes cerebrovasculares, de los cuales 4 fueron isquémicos y 3 hemorrágicos (tabla 3 ).

Entre los casos de mortalidad se encontró que un $80 \%$ eran mayores de 65 años y un $90 \%$ tenían alteraciones
Tabla 2 Resultados obtenidos en el puntaje de riesgo OESIL

\begin{tabular}{ll}
\hline Puntaje de riesgo OESIL & $\mathrm{n}(\%)$ \\
\hline Variables clínicas & \\
Edad mayor de 65 años & $88(54.66)$ \\
Historia de enfermedad cardiovascular & $57(35.40)$ \\
Ausencia de pródromos & $63(39.13)$ \\
ECG anormal & $130(80.75)$ \\
Puntaje OESIL & \\
0 & $14(8.70)$ \\
1 & $31(19.25)$ \\
2 & $58(36.02)$ \\
3 & $41(25.47)$ \\
4 & $17(10.56)$ \\
Clasificación de riesgo según puntaje de riesgo OESIL \\
Bajo (0 a 1) & $45(27.95)$ \\
Alto (2 a 4) & $116(72.05)$ \\
\hline
\end{tabular}

ECG: electrocardiograma.

Tabla 3 Incidencia acumulada de eventos adversos a los 6 meses

\begin{tabular}{ll}
\hline Evento & $\mathrm{n}(\%)$ \\
\hline Muerte & $10(6.21)$ \\
IAM & $22(13.66)$ \\
Reingresos hospitalarios & $31(19.62)$ \\
Intervenciones invasivas cardiovasculares (50) & $42(26.08)$ \\
$\quad$ Implante de marcapasos & $15(9.31)$ \\
Implante de CDI & $6(3.72)$ \\
Implante de CRT & $1(0.62)$ \\
Revascularización & $6(3.72)$ \\
Cirugía valvular & $4(2.48)$ \\
Ablación & $2(1.24)$ \\
ICP & $16(9.93)$ \\
Eventos neurológicos & $7(4.34)$ \\
ECV isquémico & $4(2.48)$ \\
Hemorragia & $3(1.86)$ \\
AIT & $0(0)$ \\
\hline
\end{tabular}

AIT: accidente isquémico transitorio; CDI: cardiodesfibrilador implantable; CRT: cardiorresincronizador; EVC: evento cerebrovascular; IAM: infarto agudo de miocardio; ICP: intervención coronaria percutánea.

electrocardiográficas, mientras que entre los pacientes que requirieron procedimientos invasivos solo el $38 \%$ tenían una edad mayor de 65 años, pero había una alta prevalencia de alteraciones electrocardiográficas en el $90 \%$. El $100 \%$ de los pacientes que reingresaron por síntomas similares tenían un electrocardiograma anormal. El 90\% $(9 / 10)$ de los pacientes que fallecieron fueron clasificados por el puntaje de riesgo OESIL como de alto riesgo, al igual que el $74 \%(23 / 31)$ de los reingresos y el $80 \%(40 / 50)$ de las intervenciones invasivas.

Para la determinación de las características operativas se calculó la sensibilidad, la especificidad, el valor predictivo positivo y el valor predictivo negativo de cada una de las posibles puntuaciones de OESIL para la detección de mortalidad, reingresos, intervenciones invasivas y la combinación de los anteriores desenlaces a 6 meses. Para mortalidad se encontró que un puntaje OESIL mayor o igual 2 tiene una sensibilidad del $90 \%$, una especificidad del $29 \%$, un valor 
Tabla 4 Sensibilidad, especificidad y valores predictivos del puntaje de riesgo OESIL con 2 o más puntos para los diferentes desenlaces a los 6 meses

\begin{tabular}{lllll}
\hline Característica & \multicolumn{4}{c}{ Desenlaces } \\
\cline { 2 - 5 } & Mortalidad & Intervenciones & Reingresos & Combinado \\
\hline Sensibilidad, \% (IC 95\%) & $90.0(55.5-99.7)$ & $81(65.9-91.4)$ & $74.19(55.4-88.1)$ & $75.76(63.5-85.5)$ \\
Especificidad, \% & 29.14 & 31.1 & 29.13 & 30.53 \\
Valor predictivo positivo, \% (IC 95\%) & $7.76(3.61-14.2)$ & $29.3(21.2-38.5)$ & $20.4(13.4-29)$ & $43.1(33.3-49.9)$ \\
Valor predictivo negativo, \% (IC 95\%) & $97.8(88.2-99.9)$ & $82.2(67.9-92)$ & $82.2(67.9-92)$ & $64.4(35.1-87.2)$ \\
\hline
\end{tabular}

IC 95\%: intervalo de confianza al 95\%.

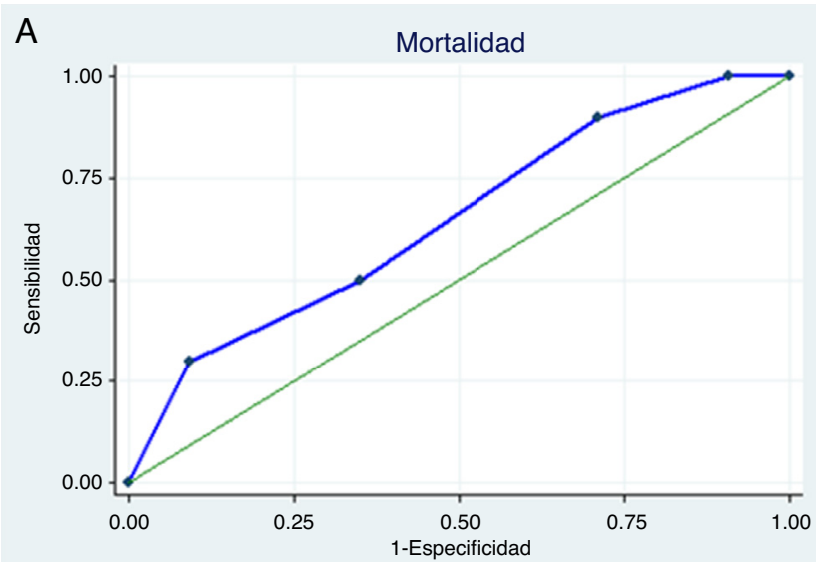

Area under ROC curve $=0.6490$

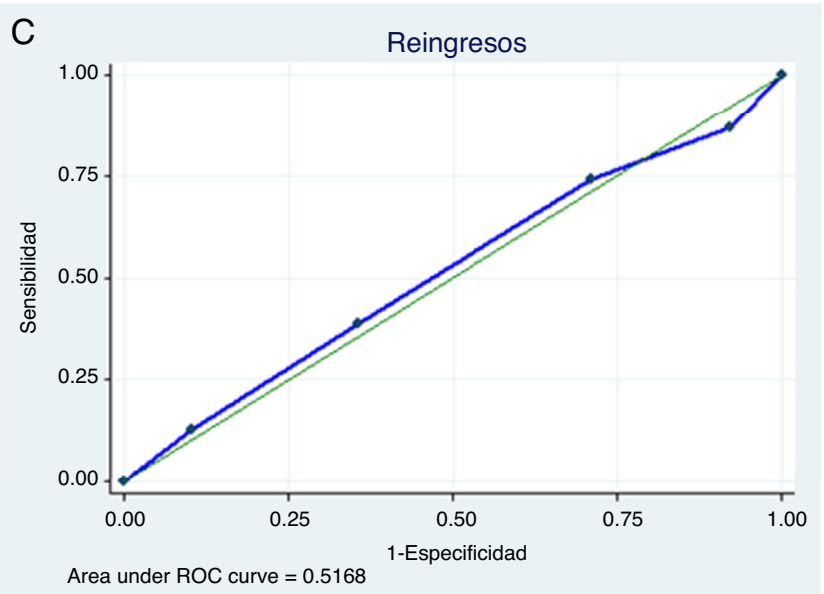

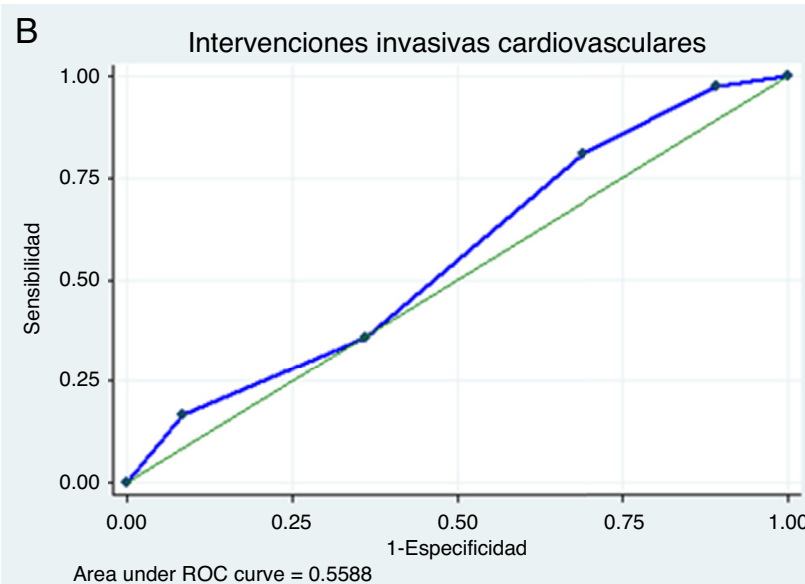

Area under ROC curve $=0.5588$

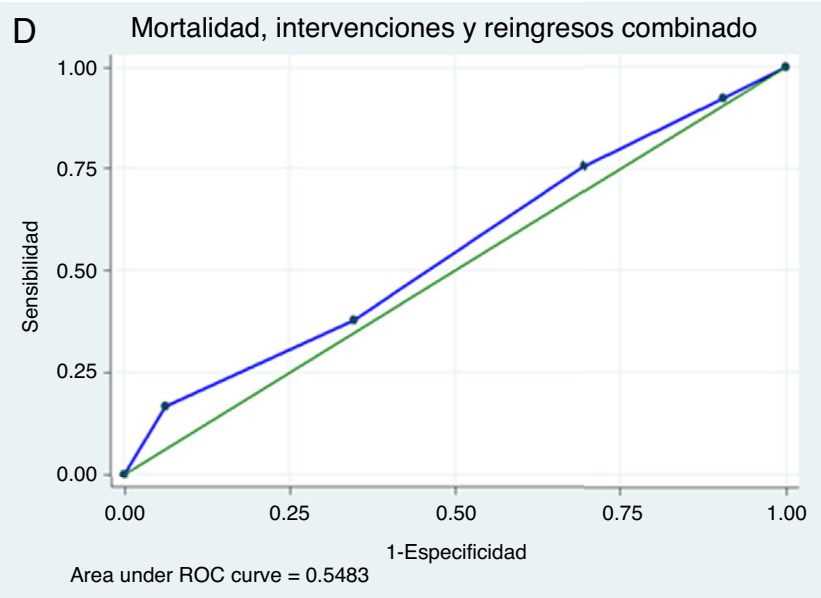

Figura 1 Curvas ROC y área bajo la curva del puntaje de riesgo OESIL.

predictivo positivo del $7.76 \%$ y un valor predictivo negativo del $\mathbf{9 7 . 8 \%}$. Para intervenciones invasivas cardiovasculares los valores fueron $81,31,29.3,82 \%$, respectivamente. Para reingresos por síncope los valores fueron 74, 29, 20, 82\%. Finalmente, para desenlaces graves combinados los datos fueron 75.7, 30.5, 43.1 y $64.4 \%$ (tabla 4 ).

Para la visualización del rendimiento global de la escala con los diferentes puntos de corte se construyeron ROC y se calculó el área bajo la curva $(A B C)$ para cada uno de los desenlaces, evidenciándose un mejor desempeño de la escala pronóstica para predecir mortalidad al tener un mayor $A B C(0.64)$ en comparación con los demás eventos (fig. 1).

\section{Discusión}

Es un reto para los médicos generales, internistas, cardiólogos y urgenciólogos predecir qué pacientes que ingresan a urgencias por síncope van a presentar un evento grave a corto o largo plazo y decidir a qué pacientes ingresar para observación y realización de pruebas e intervenciones adicionales. Esta estratificación es posible hacerla con el juicio clínico o con la utilización de herramientas de clasificación, que para síncope se han desarrollado varias, todas con inconsistencias en los subsecuentes estudios de validación y aplicación en poblaciones diferentes a la de los estudios de derivación. 
En este estudio se determinaron las características operativas de OESIL para la predicción de eventos graves a 6 meses de cada uno de los posibles puntajes en la escala, con énfasis en la clasificación de alto y bajo riesgo según si se tienen 2 o más puntos (alto riesgo), en cuyo caso encontramos una sensibilidad del $75.8 \%$ y una especificidad del $30.53 \%$ para desenlaces compuestos. Estos resultados evidencian una sensibilidad similar a la encontrada por Dipaola et al. en el 2010 y Costantino et al. en 2008; sin embargo, hay una diferencia importante en la especificidad, siendo mucho menor en nuestra población (59-60 vs. $30.5 \%)^{18,19}$. En la evaluación de desenlaces aislados, encontramos que el rendimiento para predecir mortalidad es mucho mejor en nuestra población, con una sensibilidad del $90 \%$ y un valor predictivo positivo del $97 \%$. Cabe aclarar que los estudios antes mencionados hacen la determinación pronóstica a un corto plazo (10 días) y en la literatura no hay estudios que validen este puntaje de riesgo a largo plazo (más allá de 3 meses) como se hizo en los estudios de derivación y validación inicial realizados por Colivicchi et al. con seguimiento a un año ${ }^{14}$. El estudio de comparación de escalas hecho por Reed et al. en el Reino Unido en el 2007 realizó un seguimiento a 3 meses tomando como punto de corte un puntaje de 1 ; halló una sensibilidad del $91 \%$, similar a la encontrada en nuestra población $(92.4 \%)$ con el mismo puntaje OESIL; sin embargo, la especificidad en nuestro estudio es mucho menor, con un $9.47 \%$ frente al $49 \%$ del estudio de Reed et al. ${ }^{20}$. Consideramos una fortaleza del presente estudio el seguimiento a un plazo de tiempo mucho más prolongado y más cercano al de los estudios originales, además de evaluar el rendimiento para cada uno de los posibles puntajes de la escala de riesgo.

Aunque el comportamiento del puntaje de riesgo OESIL presenta una sensibilidad similar a la de literatura externa, esta deja a un importante porcentaje de pacientes excluidos y en riesgo de eventos graves ${ }^{21-23}$. En nuestra población la especificidad es mucho más baja, indicando que un número importante de pacientes clasificados como de alto riesgo en realidad no van a presentar eventos graves y posiblemente no requieran ingreso hospitalario ni estudios adicionales. Encontramos que el valor predictivo negativo en este estudio es la característica más significativa, entendiéndose que obtener un puntaje de OESIL de 0 o 1 disminuye considerablemente la probabilidad de complicaciones y eventos graves a 6 meses.

En términos generales, el desempeño global de la prueba para predicción de reingresos, intervenciones invasivas y el desenlace combinado es limitado, demostrado por un $A B C$ muy cercano a 0.5 , con una relación casi lineal evidente en las curvas ROC. La situación es un poco más alentadora para la discriminación del riesgo de mortalidad, con un $A B C$ mayor.

Al revisar el estudio original de derivación y validación de la escala de riesgo OESIL publicado por Colivicchi et al. en $2003^{14}$, encontramos que el $A B C$ en las curvas ROC para mortalidad es mucho mayor, otorgándole más poder discriminatorio a la escala tanto en la población de derivación $(A B C 0.897)$ como en la de validación ( $A B C$ 0.894). Es posible que la discrepancia con nuestros resultados esté en asociación con las características de los grupos poblacionales, siendo nuestra población de más edad, con mediana de 67 años y media de 63 años en comparación con los
59.5 años de la cohorte de derivación y los 57 años de la cohorte de validación; así mismo, en nuestro estudio hay una mayor proporción de adultos mayores de 65 años (55 vs. $45 \%$ en derivación), una mayor proporción de enfermedad cardiovascular (35 vs. 29 y 33\%) y un mayor porcentaje de alteraciones electrocardiográficas (81 vs. 31 y 35\%).

Al igual que en los estudios de determinación de los principales factores de riesgo para mortalidad y complicaciones graves por síncope, y los estudios que validan otras escalas (San Francisco, Martin Pittsburg y EGSYS), en nuestro estudio sigue siendo consistente la presencia de alteraciones electrocardiográficas, que se demostraron en todos los casos de mortalidad y reingresos en relación con síncopes de origen cardiogénico ${ }^{9,10,22-28}$.

Aunque no fue el objetivo de este estudio, sí observamos que la proporción de complicaciones asociadas a traumatismo de cualquier tipo, incluyendo escoriaciones, fracturas y trauma de tejidos blandos posterior al episodio de pérdida de conciencia, es menor que la encontrada en la literatura mundial $(21.7 \text { vs. } 35 \%)^{4}$, pero mayor que la evidenciada en las cohortes originales de Colivicchi et al. (15\% en la cohorte de derivación y $18 \%$ en la de validación) ${ }^{14}$.

Consideramos que, además de la evaluación de desenlaces a un plazo más prolongado, nuestro trabajo tiene como fortaleza adicional ser el primero en evaluar el rendimiento pronóstico del puntaje de riesgo OESIL en población colombiana con información útil para los médicos trabajadores de los servicios de urgencias, y sus resultados pueden ser comparados con el juicio clínico y otras escalas que deben ser investigadas en nuestra población. Según nuestro conocimiento, esta es la primera validación en Latinoamérica del puntaje de riesgo OESIL con un seguimiento a largo plazo, en una población con síncope que asiste al servicio de urgencias.

Una de las principales limitaciones, propia de los estudios observaciones de cohortes, es el posible sesgo por errores de recordación de los eventos en el trascurso de los 6 meses de seguimiento por parte de los pacientes. Sin embargo, como estos no sabían si estaban clasificados en alto o bajo riesgo, según la escala, es posible que este sesgo no se presentara de manera diferencial entre los 2 grupos de pacientes; además, la mayoría de los desenlaces fueron variables objetivas, por lo que los resultados obtenidos no se ven afectados por este sesgo.

\section{Conclusiones}

Una puntuación de 2 o más en el puntaje de riesgo OESIL aplicado a población colombiana tiene un desempeño global limitado para la predicción de eventos graves (combinado de mortalidad, reingresos y procedimientos invasivos cardiovasculares) que no permitiría la adecuada discriminación de pacientes en riesgo. Sin embargo, al evaluar de forma aislada el desenlace de mortalidad en nuestra población, el rendimiento del puntaje de riesgo es mejor al presentar una sensibilidad del $90 \%$ y un valor predictivo negativo del $97.8 \%$, que orientan a un riesgo mínimo de eventos en aquellos sujetos clasificados como de bajo riesgo (puntaje menor de 2). La especificidad y el valor predictivo positivo para todos los desenlaces, tanto aislados como combinados, 
no permiten identificar a los pacientes que se benefician de ingreso hospitalario y estudios adicionales.

\section{Responsabilidades éticas}

Protección de personas y animales. Los autores declaran que para esta investigación no se han realizado experimentos en seres humanos ni en animales.

Confidencialidad de los datos. Los autores declaran que han seguido los protocolos de su centro de trabajo sobre la publicación de datos de pacientes.

Derecho a la privacidad y consentimiento informado. Los autores declaran que en este artículo no aparecen datos de pacientes.

\section{Financiación}

Universidad Nacional de Colombia.

\section{Conflicto de intereses}

Los autores declaran no tener ningún conflicto de intereses.

\section{Bibliografía}

1. Moya A, Sutton R, Ammirati F, et al. Guidelines for the diagnosis and management of syncope (version 2009). Task Force for the Diagnosis and Management of Syncope; European Society of Cardiology (ESC); European Heart Rhythm Association (EHRA); Heart Failure Association (HFA); Heart Rhythm Society (HRS). Eur Heart J. 2009;30:2631-71.

2. Alshekhlee A, Shen WK, Mackall J, et al. Incidence and mortality rates of syncope in the United States. Am J Med. 2009;122:181-8.

3. Sun BC, Emond JA, Camargo CA Jr. Characteristics and admission patterns of patients presenting with syncope to US emergency departments, 1992-2000. Acad Emerg Med. 2004;11:1029-34.

4. Benditt DG, Adkisson WO. Approach to the patient with syncope. Venues, presentations, diagnoses. Cardiol Clin. 2013;31:9-25.

5. Blanc JJ. Syncope: Definition, epidemiology, and classification. Card Electrophysiol Clin. 2013;5:387-91.

6. Quinn J, McDermott D, Stiell I, et al. Prospective validation of the San Francisco Syncope Rule to predict patients with serious outcomes. Ann Emerg Med. 2006;47:448-54.

7. Grossman SA, Fischer C, Lipsitz LA, et al. Predicting adverse outcomes in syncope. J Emerg Med. 2007;33:233-9.

8. Reed MJ, Newby DE, Coull AJ, et al. The ROSE (Risk Stratification of Syncope in the Emergency Department) study. J Am Coll Cardiol. 2010;55:713-21.

9. Puppala VK, Dickinson O, Benditt DG. Syncope. Classification and risk stratification. J Cardiol. 2014;63:171-7.

10. Costantino G, Furlan R. Syncope risk stratification in the Emergency Department. Cardio Clin. 2013;31:27-38.
11. Thiruganasambandamoorthy V, Hess EP, Alreesi A, et al. External validation of the San Francisco syncope rule in the Canadian setting. Ann Emerg Med. 2010;55:464-72.

12. Del Rosso A, Ungar A, Maggi R, et al. Clinical predictors of cardiac syncope at initial evaluation in patients referred urgently to a general hospital: The EGSYS score. Heart. 2008;94: 1620-6.

13. Mora G, Rendón I, Martínez JL, et al. Síncope y escalas de riesgo: ¿Qué evidencia se tiene? Rev Colomb Cardiol. 2011;18:330-9.

14. Colivicchi F, Ammirati F, Melina D, et al. Development and prospective validation of a risk stratification system for patients with syncope in the emergency department: The OESIL risk score. Eur Heart J. 2003;24:811-9.

15. Thygesen K, Alpert J, Jaffe A. Third universal definition of myocardial infarction. ESC/ACCF/AHA/WHF Expert Consensus Document. Circulation. 2012;126:2020-203.

16. Flahault A, Cadilhac M, Thomas G. Sample size calculation should be performed for design accuracy in diagnostic test studies. J Clin Epidemiol. 2005;58:859-62.

17. Chu H, Cole SR. Sample size calculation using exact methods in diagnostic test studies. J Clin Epidemiol. 2007;60:1201-2.

18. Dipaola F, Costantino G, Perego F, et al. San Francisco Syncope Rule, Osservatorio Epidemiologico sulla Sincope nel Lazio risk score, and clinical judgment in the assessment of short-term outcome of syncope. Am J Emerg Med. 2010;28:432-9.

19. Costantino G, Perego F, Dipaola F, et al. Short- and long-term prognosis of syncope, risk factors, and role of hospital admission: Results from the STePS (Short-Term Prognosis of Syncope) study. J Am Coll Cardiol. 2008;51:276-83.

20. Reed M, Newby DE, Coull AJ, et al. The risk stratification of syncope in the emergency department (ROSE) pilot study: A comparison of existing syncope guidelines. Emerg Med J. 2007;24:270-5.

21. Ammirati F, Colivicchi F, Santini M. Diagnosing syncope in clinical practice. Implementation of a simplified diagnostic algorithm in a multicenter prospective trial-The OESIL 2 study (Osservatorio Epidemiologico della Sincope nel Lazzio). Eur Heart J. 2000;21:935-40.

22. Hing R, Harris R. Relative utility of serum troponin and the OESIL score in syncope. Emerg Med Australas. 2005;17:31-8.

23. Numeroso F, Mossini G, Spaggiari E, et al. Syncope in the emergency department of a large northern Italian hospital: Incidence, efficacy of a short-stay observation ward and validation of the OESIL risk score. Emerg Med J. 2010;27:653-8.

24. Martin TP, Hanusa BH, Kapoor WN. Risk stratification of patients with syncope. Ann Emerg Med. 1997;29:459-66.

25. Sarasin FP, Hanusa BH, Perneger T, et al. A risk to predict arrhythmias in patients with unexplained syncope. Acad Emerg Med. 2003;10:1312-7.

26. Sun BC, Derose SF, Liang LJ, et al. Predictors of 30-day serious events in older patients with syncope. Ann Emerg Med. 2009;54:769-78.

27. Benditt DG, Can I. Initial evaluation of "syncope and collapse" the need for a risk stratification consensus. J Am Coll Cardiol. 2010;55:722-4.

28. Quinn JV, Stiell IG, McDermott DA, et al. Derivation of the San Francisco Syncope Rule to predict patients with short-term serious outcomes. Ann Emerg Med. 2004;43:224-32. 